\title{
Out with the old and in with the new: time to rethink twentieth century chemotaxonomic practices in bacterial taxonomy
}

\author{
Peter Vandamme ${ }^{1, *}$ and lain Sutcliffe $e^{2, *}$
}

\begin{abstract}
Chemotaxonomic methods played an important role in the development of the polyphasic approach to classification of Archaea and Bacteria. However, we here argue that routine application of these methods is unnecessary in an era when genomic data are available and sufficient for species delineation. Thus, authors who choose not to utilize such methods should not be forced to do so during the peer review and editorial handling of manuscripts describing novel species. Instead, we argue that chemotaxonomy will thrive if improved analytical methods are introduced and deployed, primarily by specialist laboratories, in studies at taxonomic levels above the characterisation of novel species.
\end{abstract}

Amid impressive progress in microbiome and ecological research, prokaryotic systematics appears increasingly isolated from mainstream microbiology and arguably resistant to innovation [1-3]. It is part of the tradition of gentle selfmockery among taxonomists to state that millennia will be required if we keep on describing novel bacterial species the way we do it now $[4,5]$. Nevertheless, change is evidently needed as, to date, fewer than 20000 prokaryotic species names have been validly published, and millions of species need naming [5]; whilst there is no need to be able to name every single bacterial species, it would be highly desirable to name a far more representative selection. Even though a major proportion of environmental micro-organisms remain as yet uncultivated [6], it is apparent that we already have straightforward cheap procedures sufficient to isolate numerous novel micro-organisms, whilst novel strategies are emerging [7]. Today, there is a tremendous interest in microbiome diversity and function, and there is a general lack of reference cultures for taxa which all too often are likely to be cultivable, as well illustrated by the success of 'culturomics' approaches [8]. Large numbers of potentially important bacteria remain to be formally classified with reference strains made available. For example, a recent Nature Biotechnology study [9] highlighted that over $60 \%$ of 4644 prokaryotic species in the human gut microbiome represented novel species that lacked representation in international reference databases. The same study reported that for $>80 \%$ of the prokaryotic species in the human gut microbiome there were no cultivated isolates.
The adoption and further development of high throughput cultivation methods $[7,8]$ should help systematists work at an increased scale but impediments will remain unless the taxonomic community also adopts new approaches to the publishing of such studies [5]. For instance, in a recent study, Qi et al. analysed about 3400 forest soil isolates which were dereplicated into 258 MALDI-TOF mass spectrometry-defined independent strains [10]. While subsequent publications reported three novel Pedobacter and six novel Paenibacillus species $[11,12]$, near full length $16 \mathrm{~S}$ rRNA sequence analyses revealed dozens of additional novel species and genera in this collection [10]. A major reason why only nine novel species from this collection have been formally named so far is the frequently encountered insistence of journal editors that authors provide 'polyphasic' taxonomic descriptions that are as comprehensive as possible and that include multiple types of chemotaxonomic data, reflecting recommendations from a decade ago [13]. Here we argue that this latter, specialized, subset of the phenotypic data that can be collected on novel strains (such as fatty acid, polar lipid, polyamine, carbohydrate, quinone and peptidoglycan composition data) is now largely redundant in routine species descriptions.

In contrast to nomenclature, the classification and characterization of prokaryotes is an area that is not formally regulated by the International Code of Nomenclature of Prokaryotes [14], Principle 1.4 of which protects 'freedom of taxonomic thought or action'. The use of polyphasic analyses in bacterial

Author affiliations: 'Laboratory of Microbiology, Department of Biochemistry and Microbiology, Ghent University, Ghent, Belgium; ${ }^{2}$ Northumbria University, Faculty of Health \& Life Sciences, Newcastle Upon Tyne, Tyne \& Wear, U.K.

*Correspondence: Iain Sutcliffe, iain.sutcliffe@northumbria.ac.uk; Peter Vandamme, peter.vandamme@ugent.be

Keywords: bacterial taxonomy; genome sequence; polyphasic taxonomy; chemotaxonomy.

Dedicated to This paper is dedicated to the memory of the late Professor David Minnikin, in recognition of his important role in the development and application of methods for lipid analysis in the 'golden era' of chemotaxonomy [29].

005127 (c) 2021 The Authors 
taxonomy was advocated in the 1990s [15] because, some 25 years ago, it was felt that an integrated approach was the best way to approximate the genomic information that bacteria carry, and so a proposed classification should be the best possible synthesis of as much information as possible. Since then, this has become the standardized approach to microbial taxonomy [13]. However, with the new millennium, bacterial genome sequencing gradually became more affordable and is now established in taxonomic practice [16, 17].

In essence, this is why we have argued against the strict adherence to a rigid definition of polyphasic taxonomy in the twenty-first century and in favour of a revitalization of bacterial taxonomy through an emphasis on genomics $[1-3,5]$. While Tindall et al. [13] intended to review the key elements to be considered when prokaryotes are characterized, 'with a view to providing an overview of some of the pitfalls commonly encountered in taxonomic papers', their paper has subsequently been used as a 'carved in stone'checklist for 'go/no-go' editorial decisions, thus turning a milestone paper into a millstone around taxonomists' necks. In particular, the entreatment by Tindall et al. [13] that strains 'should be characterized as comprehensively as possible' is arguably invalidated by the vastness of the microbial world; put somewhat bluntly, not all prokaryotic species will be of such individual interest as to warrant 'comprehensive' characterisation at the time of description, especially those joining the large number of genera with $\geq 10$ members already (currently 340 , approaching $10 \%$ of the genera with validly published names; https:// lpsn.dsmz.de/text/largest-genera, accessed 30/09/2021). A more pragmatic recommendation is that many strains should be characterized 'enough' to allow their discrimination from related taxa and future identification (a return to 'diagnosis' rather than 'description' [18]). Then strains deemed of interest (for example, following interrogation of their genome sequence) can be subsequently revisited for more extensive characterization.

In this context, it is appropriate to reassess the purpose and utility of chemotaxonomic data. While the International Journal of Systematic and Evolutionary Microbiology - and other taxonomic journals - does not have an explicit policy mandating the inclusion of chemotaxonomic data (www. microbiologyresearch.org/journal/ijsem/scope) in novel species descriptions, editors and peer reviewers in daily practice often behave differently. Authors clearly do not have freedom to include only those technical approaches which they deem taxonomically useful and sufficient for their descriptions. Part of this problem is that peer reviewers of taxonomic papers appear unfamiliar with the rules of prokaryotic nomenclature (notably Principle 1.4 quoted above) and that editors of these journals likewise do not correct inappropriate requests such as it is obligatory to do fatty acid, carbohydrate, quinone and peptidoglycan analyses when you report a novel species', it is obligatory to do DNA fingerprinting when reporting a novel species' or 'you must provide more important chemotaxonomic data, such as peptidoglycan type, whole-cell sugars, polar lipids, respiratory quinone and cellular fatty acids' (all were copied from recent decision letters, italics added for emphasis).

Despite a longstanding concern that 'few scientists now have extensive experience' (i.e. the requisite skills) for the 'interpretation of chemotaxonomic data' [13], editors still insist on authors providing multiple types of chemotaxonomic analyses that have, in bacterial taxonomy, essentially descriptive value only. In the classic taxonomic trinity of classification, identification and nomenclature [19], the practical value of chemotaxonomic data today is virtually non-existent for species delineation, when compared to the taxonomic resolution of phylogenomic methods. Mostly, what is confirmed by traditional chemotaxonomy is that the properties of a new member of genus $\mathrm{X}$ are very similar/ identical to those of known members of genus X (information that could have been confidently predicted from the phylogenetic placement of the new species in genus $\mathrm{X}$ !). Indeed, in some cases, the phylogenomic approach is needed to resolve ambiguities introduced by chemotaxonomy [20]. Moreover, chemotaxonomic data is often laborious to acquire (typically requiring some combination of extraction, purification, derivatization and analysis of cellular constituents) and thus relatively expensive, diverting scarce resources from more informative work. This expense is compounded by the need for parallel testing of novel strains and references strains in order to compensate for the fact that several standard chemotaxonomic characters are notably affected by changes in growth conditions, thus undermining the ability to directly compare data from the literature and demanding stringent intra-laboratory standardization, which is rarely documented. Inter-laboratory standardization is yet more difficult still and the 'manual' nature of many traditional methods has resisted the introduction of automation and the accompanying benefits of robust quality control processes.

Whilst chemotaxonomic data can be of value, it is clearly better employed in studies at taxonomic ranks above the species level. Knowledge of cellular components such as lipids and cell walls may be, or become, valuable with increasing insights into - for instance - microbe-host interactions. However, the twentieth century analytical tools employed by many taxonomists need to be replaced in such studies by much higher-resolution technologies to be truly informative. For example, the resolution of two-dimensional thin-layer chromatography, which remains routinely used in taxonomic polar lipid studies, falls far short of contemporary mass spectrometry lipidomic methods, which can resolve the few spots observed (but often not identified) on thin-layer chromatography plates into multiple lipid species (for a very clear example, see the resolution of seven bulk lipid classes into $>60$ individual lipids by Rashid et al. [21]). Similarly, the application of the thin-layer chromatography to sugar profiling of whole-cell hydrolysates remains a crude broad brushstroke approach compared to the resolution of metabolomic fingerprinting. Even fatty acid analyses, which have benefited greatly from the standardization provided by commercial diagnostic systems, still face challenges when comparing datasets between laboratories and over time. 
Collaborations with analytical chemists to introduce stateof-art methodologies are thus needed to revitalize the chemotaxonomic toolkit. Indeed, it is possible to consider a future for chemotaxonomy where the application (and re-application, and re-application...) of a checklist of entrenched methodologies in separate single species descriptions is, instead, replaced by comprehensive, informative surveys carried out in specialized laboratories. For example, Schumann et al. [22] recently applied what they themselves described as 'perceived ... "occult" methods' to re-evaluate chemotaxonomic characteristics of three type strains in the family Ruaniaceae: reanalysis using state-of-the-art methods allowed correction of the peptidoglycan structural data for two type strains, and clarification of the menaquinone profile of one of them. In addition, they identified ribose as the major whole-cell sugar in three type strains previously reported to contain various sugars other than ribose. Ironically, such 'synoptic' studies were more common when chemotaxonomy was first introduced in the 1970s: it is time to revive the practice (as we argued more than a decade ago [23]). Such studies would renew appreciation of the utility of appropriately selected chemotaxonomic data, particularly at taxonomic ranks above the species. Moreover, collaboration between bioinformaticians and chemotaxonomists should allow the further development of biosynthetic/metabolic pathway analyses into 'in silico chemotaxonomy' approaches [20, 23, 24]. Further development of spectral fingerprinting methods should also help overcome an intrinsic lack of 'portability' of data acquired from twentieth century chromatographic techniques and are likely to involve safer, 'greener' protocols than the often hazardous older methods. In this regard, it is notable that the one type of chemotaxonomic information that has practical value in daily diagnostic microbiology today is MALDI-TOF mass spectrometry analysis [25]. Novel species that have a MALDI-TOF mass spectrometry profile that is different from that of their nearest neighbours can be recognized by countless microbiologists who use MALDI-TOF mass spectrometry technology on a daily basis for diagnostic purposes by simply adding a reference spectrum to their mass spectrum library. Undoubtedly, it would significantly benefit the systematics community and further drive the adoption of this technology if freely accessible and iterative spectral database(s) were made available.

The common practice of single-strain-species descriptions resulted in an 'assembly line' of formulaic papers [26], a situation reinforced by peer reviewers and/or editors who propagate the same dogmatic adherence to the formula $[1,5]$. Traditional taxonomic journals have started to change gears and discourage publication of single-strain-species descriptions [27]. Today, editors request authors to functionally explore genome sequences or to demonstrate novel biology and features of interest in a proposed novel taxon [28]. We urge editors of taxonomic journals (and peer reviewers) to take the next step. A genome sequence suffices to prove that a strain represents a novel species or not. The request, as a conditio sine qua non, to generate multiple types of chemotaxonomic information as part of a novel species description imposes a burden that is unnecessarily high and counterproductive. It may be naive to strive to formally name all prokaryotic species, but many fields are in dire need of reference cultures that correspond to sequences that have been detected through cultivation independent analyses. We argue that a genome sequence analysis, a basic characterization of growth characteristics and the public deposit of type strains should suffice for proposing novel species. Beyond this, authors should have freedom to include (extensive) phenotypic and chemotaxonomic testing, or not, as they deem appropriate.

Funding Information

This research received no specific grant from any funding agency in the public, commercial, or not-for-profit sectors.

Acknowledgements

We thank the peer reviewers for constructive comments that improved the final text.

Author contributions

Both authors contributed to writing the original draft and subsequent review and editing.

Conflicts of interest

The authors declare that there are no conflicts of interest.

References

1. Sutcliffe IC. Challenging the anthropocentric emphasis on phenotypic testing in prokaryotic species descriptions: rip it up and start again. Front Genet 2015;6:218.

2. Sutcliffe IC, Trujillo ME, Whitman WB, Goodfellow M. A call to action for the International Committee on Systematics of Prokaryotes. Trends Microbiol 2013:21:51-52.

3. Vandamme $\mathrm{P}$, Peeters $\mathrm{C}$. Time to revisit polyphasic taxonomy. Antonie van Leeuwenhoek 2014;106:57-65.

4. Tamames J, Rosselló-Móra R. On the fitness of microbial taxonomy. Trends Microbiol 2012;20:514-516.

5. Sutcliffe IC, Rosselló-Móra R, Trujillo ME. Addressing the sublime scale of the microbial world: reconciling an appreciation of microbial diversity with the need to describe species. New Microbes New Infect 2021;43:100931.

6. Steen AD, Crits-Christoph A, Carini P, DeAngelis KM, Fierer N, et al. High proportions of bacteria and archaea across most biomes remain uncultured. ISME J 2019:13:3126-3130.

7. Lewis WH, Tahon G, Geesink P, Sousa DZ, Ettema TJG. Innovations to culturing the uncultured microbial majority. Nat Rev Microbiol 2021:19:225-240

8. Lagier J-C, Khelaifia S, Alou MT, Ndongo S, Dione N, et al. Culture of previously uncultured members of the human gut microbiota by culturomics. Nat Microbiol 2016;1:16203.

9. Almeida A, Nayfach S, Boland M, Strozzi F, Beracochea M, et al. A unified catalog of 204,938 reference genomes from the human gut microbiome. Nat Biotechnol 2021;39:105-114.

10. Qi SS, Bogdanov A, Cnockaert M, Acar T, Ranty-Roby S, et al. Induction of antibiotic specialized metabolism by co-culturing in a collection of phyllosphere bacteria. Environ Microbiol 2021;23:2132-2151.

11. Qi SS, Cnockaert M, Carlier A, Vandamme P. Pedobacter gandavensis sp. nov., Pedobacter foliorum sp. nov. and Pedobacter planticolens sp. nov., isolated from leaves of Arabidopsis thaliana. Int J Syst Evol Microbiol 2019;71:004667.

12. Qi SS, Cnockaert M, Carlier A, Vandamme PA. Paenibacillus foliorum $\mathrm{sp}$. nov., Paenibacillus phytohabitans sp. nov., Paenibacillus plantarum $\mathrm{sp}$. nov., Paenibacillus planticolens sp. nov., Paenibacillus phytorum sp. nov. and Paenibacillus germinis sp. nov., isolated from the Arabidopsis thaliana phyllosphere. Int J Syst Evol Microbiol 2021;71:004781. 
13. Tindall BJ, Rosselló-Móra R, Busse H-J, Ludwig W, Kämpfer P. Notes on the characterization of prokaryote strains for taxonomic purposes. Int J Syst Evol Microbiol 2010;60:249-266.

14. Parker CT, Tindall BJ, Garrity GM. International Code of Nomenclature of Prokaryotes Prokaryotic Code (2008 Revision). Int J Syst Evol Microbiol 2019;69:S1-S111.

15. Vandamme P, Pot B, Gillis M, de Vos P, Kersters K, et al. Polyphasic taxonomy, a consensus approach to bacterial systematics. Microbiol Rev 1996;60:407-438.

16. Chun J, Oren A, Ventosa A, Christensen H, Arahal DR, et al. Proposed minimal standards for the use of genome data for the taxonomy of prokaryotes. Int J Syst Evol Microbiol 2018;68:461-466.

17. Hugenholtz $P$, Chuvochina M, Oren A, Parks DH, Soo RM. Prokaryotic taxonomy and nomenclature in the age of big sequence data. ISME J 2021;15:1879-1892.

18. Renner SS. A return to linnaeus's focus on diagnosis, not description: the use of DNA characters in the formal naming of species. Syst Biol 2016;65:1085-1095

19. COWAN ST. Principles and practice of bacterial taxonomy-a forward look. J Gen Microbiol 1965;39:143-153.

20. Baek I, Kim M, Lee I, Na S-I, Goodfellow M, et al. Phylogeny trumps chemotaxonomy: a case study involving Turicella otitidis. Front Microbiol 2018:9:834.

21. Rashid R, Cazenave-Gassiot A, Gao IH, Nair ZJ, Kumar JK, et al. Comprehensive analysis of phospholipids and glycolipids in the opportunistic pathogen Enterococcus faecalis. PLOS One 2017;12:e0175886.

22. Schumann P, Kalensee F, Cao J, Criscuolo A, Clermont D, et al. Reclassification of Haloactinobacterium glacieicola as Occultella glacieicola gen. nov., comb. nov., of Haloactinobacterium album as Ruania alba comb. nov, with an emended description of the genus Ruania, recognition that the genus names Haloactinobacterium and Ruania are heterotypic synonyms and description of Occultella aeris sp. nov., a halotolerant isolate from surface soil sampled at an ancient copper smelter. Int J Syst Evol Microbiol 2021;71:004769.

23. Sutcliffe IC, Trujillo ME, Goodfellow M. A call to arms for systematists: revitalising the purpose and practises underpinning the description of novel microbial taxa. Antonie van Leeuwenhoek 2011;101:13-20.

24. Fotedar R, Caldwell ME, Sankaranarayanan K, Al-Zeyara A, Al-Malki A, et al. Ningiella ruwaisensis gen. nov., sp. nov., a member of the family Alteromonadaceae isolated from marine water of the Arabian Gulf. Int J Syst Evol Microbiol 2020;70:4130-4138.

25. Seng $P$, Rolain JM, Fournier PE, La Scola B, Drancourt M, et al. MALDI-TOF-mass spectrometry applications in clinical microbiology. Future Microbiol 2010;5:1733-1754.

26. Oren A, Garrity GM. Then and now: a systematic review of the systematics of prokaryotes in the last 80 years. Antonie van Leeuwenhoek 2013;106:43-56.

27. Trujillo ME, Oren A. Avoiding "salami slicing" in publications describing new prokaryotic taxa. Int J Syst Evol Microbiol 2018;68:977-978.

28. Sutcliffe IC. Valediction: descriptions of novel prokaryotic taxa published in Antonie van Leeuwenhoek-change in editorial policy and a signpost to the future? Antonie van Leeuwenhoek 2019;112:1281-1282.

29. Goodfellow M, Sutcliffe IC. In memoriam - David Ernest Minnikin (1939-2021). Int J Syst Evol Microbiol 2021;71:10.

\section{Five reasons to publish your next article with a Microbiology Society journal}

1. The Microbiology Society is a not-for-profit organization.

2. We offer fast and rigorous peer review - average time to first decision is 4-6 weeks.

3. Our journals have a global readership with subscriptions held in research institutions around the world.

4. $80 \%$ of our authors rate our submission process as 'excellent' or 'very good'.

5. Your article will be published on an interactive journal platform with advanced metrics.

Find out more and submit your article at microbiologyresearch.org. 